\title{
Green Spaces and General Health: Roles of Mental Health Status, Social Support, and Physical Activity
}

Payam Dadvand ${ }^{\mathrm{a}, \mathrm{b}, \mathrm{c}}$, Xavier Bartoll $^{\mathrm{d}, \mathrm{e}}$, Xavier Basagaña ${ }^{\mathrm{a}, \mathrm{b}, \mathrm{c}}$, Albert Dalmau-Bueno ${ }^{\mathrm{a}, \mathrm{b}, \mathrm{c}}$, David Martinez ${ }^{\mathrm{a}, \mathrm{b}, \mathrm{c}}$, Albert Ambros ${ }^{\mathrm{a}, \mathrm{b}, \mathrm{c}}$, Marta Cirach $^{\mathrm{a}, \mathrm{b}, \mathrm{c}}$, Margarita Triguero-Mas ${ }^{\mathrm{a}, \mathrm{b}, \mathrm{c}}$, Mireia Gascon ${ }^{\mathrm{a}, \mathrm{b}, \mathrm{c}, \mathrm{f}}$, Carme Borrell $^{\mathrm{b}, \mathrm{c}, \mathrm{de}}$, Mark J Nieuwenhuijsen ${ }^{\mathrm{a}, \mathrm{b}, \mathrm{c}}$

${ }^{\text {a }}$ Centre for Research in Environmental Epidemiology (CREAL), Barcelona, Spain.

${ }^{\mathrm{b}}$ Universitat Pompeu Fabra (UPF), Barcelona, Spain.

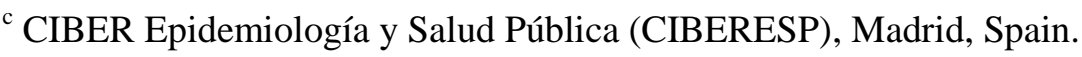

d Agència de Salut Pública de Barcelona, Barcelona, Spain.

${ }^{\mathrm{e}}$ Institut de Recerca Biomèdica Sant Pau (IIB Sant Pau), Barcelona, Spain

f ISGlobal, Barcelona Ctr. Int. Health Res. (CRESIB), Hospital Clínic - Universitat de Barcelona, Barcelona, Spain

\section{Corresponding Author:}

Payam Dadvand, CREAL, Doctor Aiguader, 88, 08003 Barcelona, Spain, [pdadvand@creal.cat], Tel +3493214 7317, Fax +34933160575 


\section{ABSTRACT}

Green spaces are associated with improved health, but little is known about mechanisms underlying such association. We aimed to assess the association between greenness exposure and subjective general health (SGH) and to evaluate mental health status, social support, and physical activity as mediators of this association. This crosssectional study was based on a population-based sample of 3461 adults residing in Barcelona, Spain (2011). We characterised outcome and mediators using the Health Survey of Barcelona. Objective and subjective residential proximity to green spaces and residential surrounding greenness were used to characterize greenness exposure. We followed Baron and Kenny's framework to establish the mediation roles and we further quantified the relative contribution of each mediator. Residential surrounding greenness and subjective residential proximity to green spaces were associated with better SGH. We found indications for mediation of these associations by mental health status, perceived social support, and to less extent, by physical activity. These mediators altogether could explain about half of the surrounding greenness association and onethird of the association for subjective proximity to green spaces. We observed indications that mental health and perceived social support might be more relevant for men and those younger than 65 years. The results for objective residential proximity to green spaces were not conclusive. In conclusion, our observed association between SGH and greenness exposure was mediated, in part, by mental health status, enhanced social support, and physical activity. There might be age and sex variations in these mediation roles. 


\section{Keywords}

Natural environment, ecosystem services, mental health, wellbeing, built environment, park 


\section{Introduction}

Contact with natural environments including green spaces has been associated with improved perceived and objective health outcomes and wellbeing (James et al. 2015). Increasing physical activity, reducing psychological stress, anxiety and depression, increasing social contacts and cohesion and reducing exposure to environmental hazard such as air pollution have been suggested as possible mechanisms for health benefits of green spaces (Dadvand et al. 2015; de Vries et al. 2013; Maas et al. 2009; Sugiyama et al. 2008). However, the available evidence investigating mediation roles of these mechanisms and their relative contributions to the observed health benefits of green spaces remains scarce. Moreover, these mediation roles might vary across strata of sex and age, but the available evidence on such a variation is non-existent. An additional issue that remains unexplored is whether the method applied to characterize greenness exposure (e.g. residential surrounding greenness, objective or subjective residential proximity to green spaces, etc.) could have implications when investigating the underlying mechanisms linking green spaces and health.

The aim of this study was to assess the association between exposure to greenness (including residential surrounding greenness and subjective and objective measures of residential proximity to green spaces) and subjective general health (SGH) and to evaluate mental health status, social support, and physical activity as potential mediators of this association, if any. As secondary aims, we also assessed relative contributions of aforementioned mediators to greenness exposure-SGH association and evaluated the variations in their mediating roles across strata of age and sex. 


\section{Methods}

\subsection{Study setting}

This cross-sectional study was based on data obtained from a population-based randomized sample of adults residing in Barcelona, Spain. The data was collected in the context of the 2011 Barcelona Health Survey aimed to study the health status, life-styles and use of health services among Barcelona residents (Bartoll et al. 2011). A face-toface interview survey by trained interviewers was conducted at the residential place of a sample of 4000 people residing across the 10 districts of Barcelona. The subjects were randomly selected from the Barcelona municipal register of residents in a way to represent the age and sex structure of districts. An invitation letter was sent to selected subjects, informing them about the objectives of the survey and asking them to participate. In total 9,969 persons were contacted to attain planned sample size of 4000 participants (i.e. response rate of $43 \%$ ). The non-responders (those who refrained from participating in the study or could not be approached after five visits to their homes) were substituted by randomly-selected persons of same district, age and sex. For this study, we limited the participants to those with age $\geq 18$ years resulting in 3461 adults being included in our analyses.

Barcelona is a port city of 1.6 million inhabitants situated on the North-eastern part of the Iberian Peninsula. It has a Mediterranean climate characterized by hot and dry summers, mild winters, and maximum precipitation and vegetation during autumn and spring. 


\subsection{Questionnaire data}

The data on the outcome (SGH), mediators (mental health status, perceived social support, and physical activity), and relevant socio-demographic covariates were obtained from the Barcelona Health Survey questionnaire.

\subsubsection{Subjective general health}

In order to characterize SGH, we asked participants: "In general, would you say that your health is..." with possible responses being: excellent/very good/good/moderate/bad (Ware Jr and Sherbourne 1992). The answers were dichotomized with cut-off at "less than good", following the same methodology used in previous studies (Maas et al. 2006; Triguero-Mas et al. 2015). We considered "less than good" answers as the reference category, therefore a positive association between greenness exposure and this variable could be interpreted as better SGH.

\subsubsection{Mental health status}

Participants answered the twelve questions of the General Health Questionnaire (GHQ12) (Goldberg 1978). GHQ-12 has been reported to be a valid and reliable tool for screening non-psychotic psychiatric problems (i.e. mental health status) in the Spanish population (Sánchez-López and Dresch 2008). The general score was dichotomized with those having a general score $\geq 3$ being classified as being at risk of psychiatric problems following the methods described by Goldberg (Goldberg 1978). We considered being at risk of psychiatric problems as the reference category; therefore, a positive association between greenness exposure and this variable could indicate better mental health status. 


\subsubsection{Perceived social support}

Participants answered the eight questions of the Duke-UNC Functional Social Support Questionnaire (FSSQ) (Broadhead et al. 1988). This questionnaire covers two dimensions of social support: the confidant support reflecting availability of people with whom one can share important life issues and concerns and receive advice and support, and emotional support characterizing expressions of love, affection, esteem, and etc. The Duke-UNC FSSQ has been shown to have an adequate validity and reliability for the Spanish population to assess social support (Bellón Saameño et al. 1996). The questionnaire was scored based on the Duke-UNC FSSQ guidelines (Bellón Saameño et al. 1996). The distribution of scores was skewed. We therefore dichotomized the score using the $75^{\text {th }}$ percentile (based on the scores of all participants) as the cut-off. We considered a score less than $75^{\text {th }}$ percentile as the reference category, therefore a positive association between greenness exposure and this variable could indicate stronger perceived social support.

\subsubsection{Physical activity}

Participants answered the seven questions of the International Physical Activity Questionnaire (IPAQ-Short version) (Craig et al. 2003). The IPAQ has been reported to have an acceptable validity and reliability for the Spanish population (Roman-Viñas et al. 2010). We developed a binary variable indicating whether the participant could be considered as having moderate or high physical activity levels based on the IPAQ guidelines (i.e. moderate/high vs. low levels of physical activity) (IPAQ Research Committee 2005). We considered low physical activity level as the reference category, 
therefore a positive association between greenness exposure and this variable could be interpreted as more likelihood of achieving moderate to high levels of physical activity.

\subsection{Greenness Exposure}

We characterized greenness exposure using three indicators: residential surrounding greenness and objective and subjective residential proximity to green spaces.

\subsubsection{Residential surrounding greenness}

Our assessment of residential surrounding greenness was based on Normalized Difference Vegetation Index (NDVI) obtained from the NASA's Earth Observing System Data and Information System (EOSDIS) website. NDVI is an indicator of greenness based on land surface reflectance of visible (red) and near-infrared parts of spectrum (Weier and Herring 2011). It ranges between -1 and 1 with higher numbers indicating more photosynthetically active greenness. We generated our NDVI map using the image obtained on 5 July 2013 by the Landsat 8 OLI (Operational Land Imager) and TIRS (Thermal Infrared Sensor) at 30m×30m resolution. Surrounding greenness was abstracted as the average of NDVI in buffers of $100 \mathrm{~m}, 250 \mathrm{~m}$, and $500 \mathrm{~m}$ (Dadvand et al. 2012a; Dadvand et al. 2012b; Dadvand et al. 2012c; Lovasi et al. 2011; Markevych et al. 2014) around the main home address of each study participant, which was geocoded according to the address where the interviews were conducted.

\subsubsection{Residential proximity to green spaces}


We used proximity to green spaces as a surrogate for access to green spaces (Expert Group on the urban environment 2001). We assessed both subjective (perceived) and objective residential proximity to green spaces.

2.3.2.1. Subjective residential proximity to green spaces: The participants answered the question (yes/no) whether they have a park within 10 minutes walk from their home.

2.3.2.2. Objective residential proximity to green spaces: To define green spaces we utilized the Parks and Garden Map of Barcelona (Parcs i Jardins de l'Ajuntament de Barcelona, 2013) developed by the Barcelona city council. Based on the European Commission recommendation on access to green spaces, we constructed a binary variable (yes/no) indicating whether the participant's home address was within $300 \mathrm{~m}$ of a major green space, defined as a green space with an area $\geq 5,000 \mathrm{~m}^{2}$ (Expert Group on the urban environment 2001).

\subsection{Mediation Analyses}

We followed the steps set by Baron and Kenny (Baron and Kenny 1986) to separately investigate the mediation roles of mental health status, perceived social support, and physical activity in the association between greenness exposure and SGH. For the residential surrounding greenness, we reported the associations for one inter-quartile range (IQR) increase in average NDVI across each buffer size based on all study participants. 
Step 1. The association between greenness exposure and SGH. We developed logistic regression models with SGH as outcome and measures of greenness exposure (one at a time) as predictor. The models were further adjusted for potential confounders identified a priori: age (18-45, 46-65, or <65), sex, and indicators of socioeconomic status (SES) at both individual and area levels. Educational attainment (no or primary/secondary/university) was used as the indicator of individual-level SES and tertiles of 2010 household income by neighbourhood (Renta Familiar Disponible) (Generalitat de Catalunya 2010) was applied as the indicator of area-level SES.

Step 2. The association between greenness exposure and mediators. We developed logistic regression models with mental health status, perceived social support, and physical activity (one at a time) as the outcome and indicators of greenness exposure (one at a time) as the predictor. All these models were further adjusted for the confounders mentioned in the step 1. For physical activity, we excluded the study participants who reported having restrictions to do physical activity.

Steps $3 \& 4$. The association between greenness exposure and mediators and SGH. We added mediators (one at a time as well as altogether) to the models described in step 1 to estimate the association between SGH and mediators controlled for greenness exposure and between SGH and greenness exposure controlled for mediators.

\subsection{Further analyses}

2.5.1. Quantifying the relative contribution of each mediator 
We used the "binary_mediation" command in STATA to obtain relative contribution of each mediator to the association between greenness exposure and SGH expressed as the proportion of the total effect that is mediated by the mediator. This command implements the Baron and Kenny's framework for mediation by fitting separately a model for the mediator as a function of exposure and a model for the outcome given the mediator and the exposure (Baron and Kenny 1986). This allows the calculation of the direct, indirect and total effects of exposure, and the proportion of effect mediated. We conducted the analyses by including all mediators in the same model to account for interrelations between the mediators. We used bootstrap to obtain percentile-based $95 \%$ confidence interval for the contribution of each mediator.

\subsubsection{Age and sex stratified analyses}

To explore potential variations in the mediation roles of our hypothesized mediators in the greenness exposure-SGH association across strata of sex and age, we stratified the aforementioned mediation analyses for age ( $<65$ years vs. $\geq 65$ years $)$ and sex. We used age of 65 as cut-off as it is the legal age of retirement in Spain which might result in a change in personal behaviour with regards to the green space use.

\section{Results}

The description of the sociodemographic characteristics of study participants, indicators of greenness exposure, outcome, and mediators are presented in Table 1. Those participants with subjective or objective residential proximity to green spaces had higher (Wilcoxon rank-sum test p-values <0.01) residential surrounding greenness in all buffer sizes (Supplementary Table S1). There was a modest agreement between 
subjective and objective measures of residential proximity to green spaces (Kappa value $=0.61, \mathrm{p}$-value $<0.01)$. As presented in Supplementary Table S2, participants with no university education, younger than 65 years, and males were more likely to live in proximity (objective) of green spaces. Subjective residential proximity to green spaces was reported more frequently by female participants and those with no university education. There was no statistically significant difference in residential surrounding greenness across strata of education, age and sex.

\subsection{The association between greenness exposure and SGH}

An increase in residential surrounding greenness was associated with better SGH, which was consistent over all buffer sizes (Table 2). Similarly, subjective residential proximity to green spaces was associated with better SGH (Table 2). Objective residential proximity to green spaces showed a non-statistically significant ( $p$-value $=0.10$ ) association with better SGH.

Further adjustment of analyses for the country of birth (low- vs. high-income countries), use of private health insurance (yes/no), and social class (manual vs. non-manual worker) did not result in a notable change in our findings. Defining objective residential proximity to green spaces based on street networks (network distance) instead of Euclidean distance used for the main analyses or relaxing the area criterion $\left(\geq 5,000 \mathrm{~m}^{2}\right)$ to identify major green spaces did not change our findings notably (Data not shown). Our findings remained unchanged after repeating the analyses using survey sampling weights for districts to account for our sampling strategy (stratified random sampling with equal allocation). 


\subsection{The association between greenness exposure and mediators}

An increase in residential surrounding greenness in all buffer sizes and subjective residential proximity to green spaces were associated with improved mental health status, stronger perceived social support, and higher likelihood of achieving moderate to high levels of physical activity (Table 2). The associations for objective residential proximity to green spaces were in the expected direction, but none attained statistical significance.

\subsection{The association between greenness exposure and mediators and SGH}

As presented in Table 3, better mental health status, enhanced perceived social support, and moderate to high levels of physical activity were associated with better SGH after adjustment (one at a time) for measures of residential surrounding greenness $(250 \mathrm{~m}$ buffer) and subjective residential proximity to green spaces. When all three mediators were included in the same model, the associations for mental health status and perceived social support remained statistically significant but physical activity lost its statistical significance (Supplementary Table 3). When all mediators were included in the model, the association for residential surrounding greenness lost its statistical significance while the association for subjective residential proximity to green spaces remained statistically significant (Supplementary Table 3).

\subsection{Relative contribution of each mediator}


We explored the relative contributions of aforementioned mediators in the associations between SGH and residential surrounding greenness (250 $\mathrm{m}$ buffer) and subjective residential proximity to green spaces for which we observed indications of mediations. Among our evaluated mediators, mental health status explained the largest part of the associations between both aforementioned indicators of greenness exposure and SGH, followed by perceived social support (Table 4). Physical activity explained the least with 95\% confidence intervals of its estimated contributions including zero (Table 4). These mediators altogether could explain half of the association between SGH and residential surrounding greenness and a third of the association with subjective residential proximity to green spaces.

\subsection{Age and sex stratified analyses}

We stratified the analyses for the associations between SGH and residential surrounding greenness (250m buffer) and subjective residential proximity to green spaces for which we observed statistically significant associations in the main analyses. Residential surrounding greenness was associated with better SGH in both sexes and age groups, but the associations were stronger and attained statistical significance only for men and those older than 65 years (Table 5). In contrast, for subjective residential proximity to green spaces, the associations were stronger and statistically significant for women and those younger than 65 years, while the associations for men and those older than 65 years were nearly statistically significant (Table 5).

In age-stratified analyses, residential surrounding greenness and subjective proximity to green spaces were associated with better mental health status and enhanced perceived 
social support only in those participants younger than 65 years (Table 5). For these participants, residential surrounding greenness was also associated with higher likelihood of achieving moderate to high physical activity levels (Table 5). In sexstratified analyses, residential surrounding greenness and subjective proximity to green spaces were associated with better mental health status and higher levels of physical activity only in men (Table 5). For perceived social support, while the associations with residential surrounding greenness were comparable for both sexes, the association with subjective residential proximity to a green space was stronger and statistically significant only for men.

As presented in Supplementary Table S3, better mental health status and stronger perceived social support were associated with better SGH in all ages and sexes, after controlling for measures of greenness exposure. Moderate to high levels of physical activity was associated with better SGH only in men and participants older than 65 years.

As presented in Supplementary Table S4, the mediators altogether could explain a larger part of the SGH-Greenness exposure associations for men and those less than 65 years. However, the confidence intervals for the contribution estimates were wide and only in men the contributions were statistically significant.

\section{Discussion}

Residential surrounding greenness and subjective residential proximity to green spaces were associated with better SGH. We found indications for mediation of these 
associations by mental health status, perceived social support, and to less extent by physical activity. These mediators appeared to be more relevant for the impact of residential surrounding greenness than subjective proximity to green spaces. We also observed some indications for variations in these mediation roles across strata of sex and age. We did not observe any association between objective residential proximity to green spaces and SGH.

\subsection{Greenness exposure and SGH}

Our observed positive association between greenness exposure and SGH is in line with findings of previous studies (Maas et al. 2009; Maas et al. 2006; Maas et al. 2008; Triguero-Mas et al. 2015). In our study, while subjective residential proximity was associated with better $\mathrm{SGH}$, the results for objective proximity to green spaces were not conclusive. We observed a modest agreement between subjective and objective proximity to green spaces, in line with findings of previous studies reporting a poor correspondence between these two measures (Ball et al. 2008; Lackey and Kaczynski 2009). Accordingly, it has been suggested that both physical presence of green spaces and how people are aware and conceptualize their presence play important roles in shaping people's behaviour regarding use of these spaces (Durand et al. 2011; Lackey and Kaczynski 2009). In this context, our findings might suggest that perceived availability of green spaces could better predict health benefits of green spaces compared with physical availability of green spaces.

We adjusted our analyses for indicators of SES at both individual (i.e. education) and area levels (neighbourhood average household income). Further adjustment of our 
analyses for other indicators of SES such as ethnicity, insurance type, and social class did not change our results notably. Moreover, while residential surrounding greenness did not differ between different SES groups, we observed beneficial associations between this measure of greenness exposure and SGH and all of our evaluated mediators. These observations might suggest that our results were unlikely to have been affected by residual SES confounding.

4.2. Mediation roles of mental health status, perceived social support, and physical activity

We found that residential surrounding greenness and subjective proximity to green spaces are associated with mental health status, perceived social support, and physical activity levels. Exposure to greenness has been associated with better mental health status (Gascon et al. 2015). Available evidence on the association between greenness exposure and social support/contact is still limited but is generally suggestive for such an association (de Vries et al. 2013; Maas et al. 2009; Sugiyama et al. 2008), with some exceptions (Triguero-Mas et al. 2015). The body of evidence on the impact of greenness exposure on physical activity is not consistent with a notable heterogeneity in the reported direction and strength of associations (Lachowycz and Jones 2011).

Our mediation analyses indicated that our observed relationship between this exposure and SGH could be mediated, in part, by mental health status and perceived social support. Our findings for a mediation role of physical activity for such an association were less solid but still suggestive for such a mediation role. The number of studies evaluating such mediation roles is still very limited. A study by de Vries et al. (2013) 
showed stress reduction and social cohesion were main mediators of the association between streetscape greenery and SGH (de Vries et al. 2013). Their findings for the mediation role of physical activity were less conclusive. While they were suggestive for a modest mediation role for the physical activity occurred in green spaces ("green physical activity"), they did not support any mediation role for the total physical activity (de Vries et al. 2013). A recent study by Triguero-Mas et al. (2015) in Spain, however, did not support such a mediation between greenness exposure and SGH by social cohesion nor by physical activity (Triguero-Mas et al. 2015). Physical activity was not a mediator of the association between neighbourhood greenness and SGH in a study by Maas et al. in the Netherlands (Maas et al. 2008). In another study, Maas et al. reported loneliness and perceived shortage of social support as mediators of the association between neighbourhood greenness and SGH (Maas et al. 2009). With regards to the association between greenness exposure and other health outcomes, a study by Sugiyama et al. (2008) found mediation roles of recreational walking and social coherence for the association between perceived neighbourhood greenness and physical health (Sugiyama et al. 2008). In a study by Richardson et al. (2008) physical activity could only explain a small part of the association between neighbourhood greenness and cardiovascular health (Richardson et al. 2013). In summary, while the available evidence supports mediation roles of mental health status and social support/cohesion for health benefits of green spaces, studies on a similar mediation role for physical activity are mainly inconclusive or suggestive for a modest mediation role.

\subsection{Relative contributions of mediators}


Among our hypothesized mediators for the association between SGH and greenness exposure, mental health status appeared to be the main mediator, followed by perceived social support. Physical activity contributed the least and the confidence intervals for its contribution included zero. In line with these findings, physical activity was not a predictor of SGH after including mental health status and perceived social support in the models.

We are aware of only one study quantifying the relative contribution of mediators to the association between greenness exposure and SGH. In that study by de Vries et al. (2013), while stress reduction and social cohesion were main mediators of the association between streetscape greenery and SGH, green physical activity contributed the least, a pattern comparable to our findings (de Vries et al. 2013). Their study showed these mediators could explain about $40 \%$ of the association in line with our findings for residential surrounding greenness that showed about half of the association between SGH and greenness exposure can be explained by these mediators. The findings of these two studies suggest that a notable part of the impact of greenness exposure on SGH could not be explained by mental health status, social support/cohesion, and physical activity and there could be other mediators involved such as reduction in exposure to environmental hazards (e.g. air pollution, noise, and heat). There might also be a potential for underestimation of the contributions of these mediators due to the misclassification in the characterisation of these mediators.

We are not aware of any available report comparing the mediation roles for the health benefits of residential surrounding greenness and residential proximity to green spaces. We observed that our hypothesized mediators altogether could explain about half of the 
association between residential surrounding greenness and SGH and make the association for residential surrounding greenness statistically non-significant; whereas, for subjective residential proximity to green spaces, these mediators could explain about one-third of the association and the association for this measures of greenness exposure remained statistically significant. These findings might suggest that mental health status, perceived social support, and physical activity are more relevant mediators for residential surrounding greenness compared to subjective residential proximity to green spaces.

\subsection{Age and sex variations in mediation roles}

We observed some indications that mental health status, social support, and physical activity as mediators might be more relevant for men. Our findings also suggested that while mental health status and social support could be more relevant mediators for participants younger than 65 years, physical activity might be more relevant for those older than 65 years.

To our knowledge, this study is the first to investigate a potential age and sex variation in mediation roles of mechanisms underlying health benefits of green spaces. It is therefore not possible to compare our findings with those of others. However, our observation is consistent with findings of previous studies showing how age and sex can affect use of green space (Lee and Maheswaran 2011). It has been suggested that elderly and teenagers are the least frequent users of green spaces (Lee and Maheswaran 2011). Also men have been reported to use green spaces more frequently and be more physically active in green spaces than women (Lee and Maheswaran 2011). These 
findings are in line with our observed more consistent patterns of mediation for male participants and those younger than 65 years. However, there is some evidence that elderly are among the population sub-groups that benefit the most from green spaces in vicinity of their homes (Maas et al. 2006).

\subsection{Limitations}

Our cross-sectional study had a limited capability for a causal inference regarding our evaluated associations and mediation roles. We applied self-reported questionnaires to obtain data on outcome and mediators of this analysis. Although we did not validate these questionnaires for our study participants, they have been shown to have proper validity and reliability for the Spanish population (Bellón Saameño et al. 1996; RomanViñas et al. 2010; Sánchez-López and Dresch 2008; Vilagut et al. 2005). Conceptually, there could have been a potential overlap between our measures of SGH and mental health status which might have resulted in overestimation of the mediation role of mental health in our analyses. Similarly, inter-individual differences in response tendencies might have influenced our analysis of the association between subjective proximity to green spaces and SGH. Our map of major green spaces did not address the quality of green spaces. Quality characteristics of green spaces like aesthetics, biodiversity, walkability, sport/play facilities, safety, and organized social events have been suggested to predict the use of green spaces (McCormack et al. 2010) and could have affected our analyses of the mediation role of physical activity, resulting in underestimation of such a role. By using an NDVI map obtained at a single point in time (2013), we effectively assumed that the spatial distribution of NDVI across our study region remained constant over the study period (2011). Our previous studies 
demonstrated a comparable variation and strong correlation between measures of greenness exposures based on NDVI maps obtained in different seasons and years and these measures also showed consistent associations with health outcomes (Dadvand et al. 2012c; Dadvand et al. 2014). Further to mental health status, social contacts, and physical activity, reduction in exposure to environmental hazards such as air pollution, noise, and heat have been proposed as potential mechanisms underlying health benefits of greenness exposure. Investigating such mediation roles was out of the scope of this study and can be tested in future studies.

\section{Conclusions}

We observed better SGH associated with residential surrounding greenness and subjective proximity to green spaces which could be explained in part by mental health status and perceived social support and to less extent, by higher levels of physical activity associated with these measures of greenness exposure. These mediators appeared to be more relevant for the impact of residential surrounding greenness than for that of subjective residential proximity to green spaces. We also observed some indications for a potential age and sex variation in these mediating roles with mental health status and perceived social support appearing to be more relevant for male participants and those younger than 65 years. Our findings for objective residential proximity to green spaces were not conclusive. Such a difference between health impacts of objective and subjective residential proximity to green spaces which has been investigated by only a handful of previous studies might be suggestive for a critical role of how people perceive and conceptualize availability of these spaces and merits further investigations. Such a role, if confirmed by future studies, could have 
important implications for policymakers when implementing policies aimed at promoting use of green spaces. We recommend future studies to apply objective measures of outcomes and mediators together with both subjective and objective measures of residential proximity to green spaces to investigate our observed associations and mediations in other settings with different climates and cultures.

Acknowledgements: This study was conducted as part of PHENOTYPE project (Positive Health Effects of the Natural Outdoor Environment in Typical Populations in Different Regions in Europe) (www.phenotype.eu) funded by the European Commission Seventh Framework Programme (Grant No. 282996). Payam Dadvand is funded by a Ramón y Cajal fellowship (RYC-2012-10995) awarded by the Spanish Ministry of Economy and Finance. Margarita Triguero-Mas is funded by a pre-doctoral grant from the Catalan Government (AGAUR FI-DGR-2013). 


\section{References}

Ball, K.,Jeffery, R.W.,Crawford, D.A., et al., Mismatch between perceived and objective measures of physical activity environments. Prev Med 2008;47:294298.

Baron, R.M.,Kenny, D.A., The moderator-mediator variable distinction in social psychological research: Conceptual, strategic, and statistical considerations. J Pers Soc Psychol 1986;51:1173-1182.

Bartoll, X.,Rodríguez-Sanz, M.,Borrell, C. 2011. [Manual de l'Enquesta de Salut de Barcelona 2011]. [accessed 1 March 2015].

Bellón Saameño, J.A.,Delgado Sánchez, A.,Luna del Castillo, J.d.D.,Lardelli Claret, P., Validez y fiabilidad del cuestionario de apoyo social funcional Duke-UNC-11. Aten Primaria 1996;18:153-163.

Broadhead, W.E.,Gehlbach, S.H.,De Gruy, F.V.,Kaplan, B.H., The Duke-UNC Functional Social Support Questionnaire: Measurement of social support in family medicine patients. Med care 1988;26:709-723.

Craig, C.L.,Marshall, A.L.,Sjöström, M., et al., International Physical Activity Questionnaire: 12-Country Reliability and Validity. Med Sci Sports Exerc 2003;35:1381-1395.

Dadvand, P.,de Nazelle, A.,Figueras, F., et al., Green space, health inequality and pregnancy. Environ Int 2012a;40:110-115. 
Dadvand, P.,de Nazelle, A.,Triguero-Mas, M., et al., Surrounding Greenness and Exposure to Air Pollution During Pregnancy: An Analysis of Personal Monitoring Data. Environ Health Perspect 2012b;120:1286-1290.

Dadvand, P.,Nieuwenhuijsen, M.J.,Esnaola, M., et al., Green spaces and cognitive development in primary schoolchildren. Proc Natl Acad Sci U S A 2015;112:7937-7942.

Dadvand, P.,Sunyer, J.,Basagaña, X., et al., Surrounding Greenness and Pregnancy Outcomes in Four Spanish Birth Cohorts. Environ Health Perspect 2012c;120:1481-1487.

Dadvand, P.,Wright, J.,Martinez, D., et al., Inequality, green spaces, and pregnant women: Roles of ethnicity and individual and neighbourhood socioeconomic status. Environ Int 2014;71:101-108.

de Vries, S.,van Dillen, S.M.E.,Groenewegen, P.P.,Spreeuwenberg, P., Streetscape greenery and health: Stress, social cohesion and physical activity as mediators. Soc Sci Med 2013;94:26-33.

Durand, C.P.,Andalib, M.,Dunton, G.F.,Wolch, J.,Pentz, M.A., A systematic review of built environment factors related to physical activity and obesity risk: implications for smart growth urban planning. Obes Rev 2011;12:e173-e182.

Expert Group on the urban environment. 2001. Towards a Local Sustainability Profile European Common Indicators. Available: http://bookshop.europa.eu/en/towardsa-local-sustainability-profile-pbKH3801996/ [accessed January 7 2016]. 
Gascon, M.,Triguero-Mas, M.,Martínez, D., et al., Mental Health Benefits of LongTerm Exposure to Residential Green and Blue Spaces: A Systematic Review. Int J Environ Res Public Health 2015;12:4354-4379.

Generalitat de Catalunya. 2010. Renta familiar disponible bruta. Available: http://www.idescat.cat/pub/?id=aec\&n=367\&m=m\&lang=es $\quad$ [accessed March 20 2013].

Goldberg, D. Manual of General Health Questionnaire. Windsor, UK: NFER publishing; 1978.

IPAQ Research Committee. 2005. Guidelines for data processing and analysis of the International Physical Activity Questionnaire (IPAQ). Available: https://docs.google.com/viewer?a=v\&pid=sites\&srcid=ZGVmYXVsdGRvbWF pbnx0aGVpcGFxfGd4OjE0NDgxMDk3NDU1YWRIZTM [accessed 5 January 2015].

James, P.,Banay, R.F.,Hart, J.E.,Laden, F., A Review of the Health Benefits of Greenness. Curr Epidemiol Rep 2015;2:131-142.

Lachowycz, K.,Jones, A.P., Greenspace and obesity: a systematic review of the evidence. Obes Rev 2011;12:e183-e189.

Lackey, K.,Kaczynski, A., Correspondence of perceived vs. objective proximity to parks and their relationship to park-based physical activity. Int J Behav Nutr Phys Act 2009;6:53.

Lee, A.C.,Maheswaran, R., The health benefits of urban green spaces: a review of the evidence. J Public Health (Oxf) 2011;33:212-222. 
Lovasi, G.,Jacobson, J.,Quinn, J., et al., Is the Environment Near Home and School Associated with Physical Activity and Adiposity of Urban Preschool Children? J Urban Health 2011;88:1143-1157.

Maas, J.,Van Dillen, S.M.E.,Verheij, R.A.,Groenewegen, P.P., Social contacts as a possible mechanism behind the relation between green space and health. Health Place 2009;15:586-595.

Maas, J.,Verheij, R.A.,Groenewegen, P.P.,De Vries, S.,Spreeuwenberg, P., Green space, urbanity, and health: how strong is the relation? J Epidemiol Community Health 2006;60:587-592.

Maas, J.,Verheij, R.A.,Spreeuwenberg, P.,Groenewegen, P.P., Physical activity as a possible mechanism behind the relationship between green space and health: a multilevel analysis. BMC Public Health 2008;8:206.

Markevych, I.,Fuertes, E.,Tiesler, C.M.T., et al., Surrounding greenness and birth weight: Results from the GINIplus and LISAplus birth cohorts in Munich. Health Place 2014;26:39-46.

McCormack, G.R.,Rock, M.,Toohey, A.M.,Hignell, D., Characteristics of urban parks associated with park use and physical activity: A review of qualitative research. Health Place 2010;16:712-726.

Richardson, E.A.,Pearce, J.,Mitchell, R.,Kingham, S., Role of physical activity in the relationship between urban green space and health. Public Health 2013;127:318324. 
Roman-Viñas, B.,Serra-Majem, L.,Hagströmer, M., et al., International Physical Activity Questionnaire: Reliability and validity in a Spanish population. Eur J Sport Sci 2010;10:297-304.

Sánchez-López, M.,Dresch, V., The 12-Item General Health Questionnaire (GHQ-12): Reliability, external validity and factor structure in the Spanish population. Psicothema 2008;20:839-843.

Sugiyama, T.,Leslie, E.,Giles-Corti, B.,Owen, N., Associations of neighbourhood greenness with physical and mental health: do walking, social coherence and local social interaction explain the relationships? J Epidemiol Community Health 2008;62:e9.

Triguero-Mas, M.,Dadvand, P.,Cirach, M., et al., Natural outdoor environments and mental and physical health: Relationships and mechanisms. Environ Int $2015 ; 77: 35-41$.

Vilagut, G.,Ferrer, M.,Rajmil, L., et al., [The Spanish version of the Short Form 36 Health Survey: a decade of experience and new developments]. Gac Sanit 2005;19:135-150.

Ware Jr, J.E.,Sherbourne, C.D., The MOS 36-item short-form health survey (SF-36): I. Conceptual framework and item selection. Med Care 1992;30:473-483.

Weier, J.,Herring, D. 2011. Measuring vegetation (NDVI \& EVI). Available: http://earthobservatory.nasa.gov/Features/MeasuringVegetation/ [accessed 17 November, 2015. 
Table 1. Description ${ }^{\text {a }}$ of sociodemographic characteristics of study participants and measures of greenness exposure.

\begin{tabular}{ll}
\hline Variable & Description \\
\hline Age & \\
$\quad 18-45$ years & $1,571(45.4 \%)$ \\
$\quad 46-65$ years & $1,039(30.0)$ \\
$\quad \geq 65$ years & $851(24.6 \%)$ \\
Sex & \\
$\quad$ Male & $1,657(47.9 \%)$ \\
$\quad$ Female & $1,804(52.1 \%)$ \\
Education & \\
$\quad$ No or primary school & $666(19.2 \%)$ \\
$\quad$ Secondary school & $1,618(46.8 \%)$ \\
$\quad$ University & $1,148(33.2 \%)$ \\
$\quad$ Missing & $29(0.8 \%)$
\end{tabular}

$\begin{array}{ll}\text { Subjective green space proximity }^{\mathbf{b}} & \\ \text { Yes } & 2,121(61.3 \%) \\ \text { No } & 817(23.6 \%) \\ \text { Missing } & 523(15.1 \%) \\ \text { Objective green space proximity }^{\mathbf{c}} & \\ \text { Yes } & 2,306(66.6 \%) \\ \text { No } & 1,155(33.4 \%)\end{array}$

$\begin{array}{ll}\text { Surrounding greenness (NDVI) } & \\ 100 \mathrm{~m} \text { buffer } & 0.18(0.06) \\ 250 \mathrm{~m} \text { buffer } & 0.19(0.05) \\ 500 \mathrm{~m} \text { buffer } & 0.20(0.05)\end{array}$

$\begin{array}{ll}\text { Subjective general health } & \\ \text { Excellent/very good/good } & 2,702(78.2 \%) \\ \text { Fair/bad } & 755(21.8 \%)\end{array}$

\section{Mental health status}

At risk

$480(14.4 \%)$

Not at risk

$2,856(85.6 \%)$

$\begin{array}{ll}\text { Physical activity } & \\ \text { Moderate/high } & 236(7.5 \%) \\ \text { Low } & 2,907(92.5 \%)\end{array}$

\section{Social support}

$\begin{array}{ll}\text { Strong } & 1,257(37.9 \%) \\ \text { Not strong } & 2,061(62.1 \%)\end{array}$

a Results are presented as count (\%) for categorical variables and as median (interquartile range) for continuous variables.

$\mathrm{b}$ The answer to the question that whether they have a park within 10 minutes walk from their home.

c Indicating whether the participant's home was located within $300 \mathrm{~m}$ of a major green space (area $\geq$ $\left.5,000 \mathrm{~m}^{2}\right)$. 
Table 2. Adjusted ${ }^{\mathrm{a}}$ odds ratio (95\% confidence intervals) of the association between measures of greenness exposure and self-subjective general health, perceived social support, mental health status, and moderate/high physical activity.

\begin{tabular}{|c|c|c|c|c|c|}
\hline & \multicolumn{3}{|c|}{ Residential surrounding greenness (NDVI) } & \multicolumn{2}{|c|}{ Residential proximity to Green spaces } \\
\hline & $100 \mathrm{~m}$ buffer & $250 \mathrm{~m}$ buffer & $500 \mathrm{~m}$ buffer & Subjective & Objective \\
\hline General Health & $1.17(1.05,1.31)^{* *}$ & $1.18(1.06,1.32)^{* *}$ & $1.16(1.05,1.29)^{* *}$ & $1.36(1.11,1.67)^{* *}$ & $1.17(0.97,1.41)$ \\
\hline Social support & $1.15(1.05,1.25)^{* *}$ & $1.17(1.08,1.28)^{* *}$ & $1.22(1.12,1.33)^{* *}$ & $1.31(1.10,1.55)^{* *}$ & $1.13(0.97,1.32)$ \\
\hline Mental health & $1.32(1.16,1.51)^{* *}$ & $1.25(1.10,1.42)^{* *}$ & $1.17(1.04,1.32)^{* *}$ & $1.30(1.04,1.63)^{* *}$ & $1.20(0.97,1.48)^{*}$ \\
\hline Physical activity & $1.20(1.03,1.41)^{* *}$ & $1.22(1.05,1.43)^{* *}$ & $1.25(1.07,1.45)^{* *}$ & $1.52(1.05,2.19)^{* *}$ & $1.33(0.96,1.83)^{*}$ \\
\hline
\end{tabular}

* p-value $<0.10$

$* *$ p-value $<0.05$

a Adjusted for age, sex, education, and neighbourhood socioeconomic status. 
Table 3. Odds ratio (95\% confidence intervals) of the association between good subjective general health and perceived social support, mental health status, and moderate/high physical activity adjusted for age, sex, education, neighbourhood socioeconomic status, and exposure to greenness (residential surrounding greenness (250 m buffer) and subjective residential proximity to green spaces).

\begin{tabular}{|c|c|c|c|c|c|}
\hline & & Greenness exposure & Social support & Mental health & Physical activity \\
\hline & & \multicolumn{4}{|c|}{ Models including residential surrounding greenness } \\
\hline \multirow[t]{3}{*}{ General health } & Single-mediator models & - & $1.79(1.46,2.19)^{* *}$ & $5.50(4.36,6.94)^{* *}$ & $1.45(1.00,2.11)^{*}$ \\
\hline & All-mediators model & $1.09(0.97,1.23)$ & $1.58(1.27,1.96)^{* *}$ & $4.94(3.87,6.30)^{* *}$ & $1.35(0.92,2.01)$ \\
\hline & & \multicolumn{4}{|c|}{ Models including subjective residential proximity } \\
\hline \multirow[t]{2}{*}{ General health } & Single-mediator models & - & $1.80(1.46,2.23)^{* *}$ & $6.23(4.86,7.99)^{* *}$ & $1.55(1.04,2.31)^{* *}$ \\
\hline & All-mediators model & $1.36(1.08,1.71)^{* *}$ & $1.51(1.20,1.90)^{* *}$ & $5.58(4.29,7.25)^{* *}$ & $1.41(0.93,2.13)$ \\
\hline
\end{tabular}

$*$ p-value $<0.10$

$* *$ p-value $<0.05$ 
Table 4. Percent (95\% confidence intervals) of the adjusted ${ }^{\mathrm{a}}$ associations between measures of greenness exposure and subjective general health explained by perceived social support, mental health status, and physical activity.

\begin{tabular}{lcc}
\hline & $\begin{array}{c}\text { Residential surrounding } \\
\text { greenness (NDVI) }\end{array}$ & $\begin{array}{c}\text { Subjective proximity to } \\
\text { green spaces }\end{array}$ \\
\hline Social support & $9.9 \%(3.3 \%, 31.4 \%)$ & $6.0 \%(1.2 \%, 16.4 \%)$ \\
Mental health & $40.0 \%(16.9 \%, 104.8 \%)$ & $19.5 \%(3.2 \%, 42.3 \%)$ \\
Physical activity & $4.3 \%(-1.1 \%, 18.5 \%)$ & $3.1 \%(-1.4 \%, 12.8 \%)$ \\
Altogether & $54.2 \%(28.8 \%, 146.8 \%)$ & $29.5 \%(10.5 \%, 59.9 \%)$ \\
\hline
\end{tabular}

a Adjusted for age, sex, education, and neighbourhood socioeconomic status. 
Table 5. Adjusted odds ratio ( $95 \%$ confidence intervals) of the association between residential surrounding greenness ( $250 \mathrm{~m}$ buffer) and subjective residential proximity to green spaces and self-subjective general health, perceived social support, mental health status, and moderate/high physical activity stratified for age and sex.

\begin{tabular}{|c|c|c|c|}
\hline Outcome & Strata & $\begin{array}{l}\text { Surrounding } \\
\text { greenness }\end{array}$ & $\begin{array}{l}\text { Subjective } \\
\text { proximity to green } \\
\text { spaces }\end{array}$ \\
\hline \multirow[t]{6}{*}{ General Health } & Age & & \\
\hline & $<65$ years $^{\mathrm{a}}$ & $1.14(1.00,1.30)^{*}$ & $1.35(1.03,1.75)^{* *}$ \\
\hline & $\geq 65$ years $^{\mathbf{b}}$ & $1.24(1.04,1.49)^{* *}$ & $1.37(0.99,1.89)^{*}$ \\
\hline & $\operatorname{Sex}^{c}$ & & \\
\hline & Male & $1.33(1.13,1.57)^{* *}$ & $1.32(0.98,1.78)^{*}$ \\
\hline & Female & $1.07(0.92,1.23)$ & $1.41(1.07,1.86)^{* *}$ \\
\hline \multirow[t]{6}{*}{ Social support } & Age & & \\
\hline & $<65$ years $^{\mathrm{a}}$ & $1.17(1.07,1.29)^{* *}$ & $1.31(1.07,1.59)^{* *}$ \\
\hline & $\geq 65$ years $^{\mathbf{b}}$ & $1.17(0.97,1.41)$ & $1.22(0.85,1.74)$ \\
\hline & $\operatorname{Sex}^{\mathrm{c}}$ & & \\
\hline & Male & $1.17(1.04,1.32)^{* *}$ & $1.54(1.20,1.98)^{* *}$ \\
\hline & Female & $1.18(1.06,1.33)^{* *}$ & $1.12(0.89,1.43)$ \\
\hline \multirow[t]{6}{*}{ Mental health } & Age & & \\
\hline & $<65$ years $^{\mathrm{a}}$ & $1.28(1.11,1.48)^{* *}$ & $1.46(1.12,1.89)^{* *}$ \\
\hline & $\geq 65$ years $^{\mathbf{b}}$ & $1.14(0.87,1.48)$ & $0.94(0.60,1.48)$ \\
\hline & $\operatorname{Sex}^{\mathrm{c}}$ & & \\
\hline & Male & $1.44(1.17,1.79)^{* *}$ & $1.43(1.01,2.02)^{* *}$ \\
\hline & Female & $1.14(0.97,1.33)$ & $1.20(0.89,1.62)$ \\
\hline \multirow[t]{6}{*}{ Physical activity } & Age & & \\
\hline & $<65$ years $^{\mathrm{a}}$ & $1.25(1.06,1.48)^{* *}$ & $1.26(0.84,1.89)$ \\
\hline & $\geq 65$ years $^{\mathbf{b}}$ & $1.07(0.78,1.46)$ & $1.69(0.91,3.17)^{*}$ \\
\hline & $\operatorname{Sex}^{\mathrm{c}}$ & & \\
\hline & Male & $1.26(1.03,1.53)^{* *}$ & $1.57(1.01,2.42)^{* *}$ \\
\hline & Female & $1.14(0.90,1.45)$ & $1.12(0.65,1.92)$ \\
\hline
\end{tabular}

$*$ p-value $<0.10$

** p-value $<0.05$

a Adjusted for age, sex, education, and neighbourhood socioeconomic status.

b Adjusted for sex, education, and neighbourhood socioeconomic status.

c Adjusted for age, education, and neighbourhood socioeconomic status. 